\title{
La dé-radicalisation en Belgique
}

\author{
André DUMOULIN*
}

La vision stratégique de la Défense belge de juin $2016^{1}$ mentionne que " le conflit syrien offre la meilleure illustration du lien entre sécurité extérieure et intérieure en raison de la participation et du retour en Europe de personnes radicalisées ayant combattu en Syrie " (p. 25). Dans le cadre de la lutte contre le terrorisme, l'accord de gouvernement du 10 octobre 2014 (p. 143 par. 6.4.5) mentionne l'approche intégrale de la radicalisation : " La protection de l'État de droit démocratique et la sécurité de nos citoyens constituent une priorité absolue pour le gouvernement. Aujourd'hui, elle se trouve sous la pression de la menace croissante de la radicalisation et du terrorisme [...]. Par conséquent, le gouvernement élaborera une approche intégrale de la radicalisation dans le cadre de laquelle une concertation sera développée à court terme avec les entités fédérées et une politique sera développée au niveau tant préventif ${ }^{2}$, proactif et judiciaire qu'administratif ".

Quel est l'état de la question plusieurs années après cette déclaration ? Quels sont les programmes de déradicalisation ${ }^{3}$ ou plutôt de désengagement ? Quelle est la politique menée par la Belgique ? Sommes-nous prêts pour assurer le suivi des " returnees " et faire face aux terroristes potentiels solitaires non décelables? Pouvons-nous intégrer le concept de " radicalisation " dont une des définitions désigne " l'adoption progressive et évolutive d'une pensée rigide, vérité absolue et non négociable, dont la logique structure la vision du monde des acteurs, qui usent pour la faire entendre de répertoires d'action violents, le plus souvent au sein de structures clandestines, formalisées ou virtuelles, qui les isolent des référents sociaux ordinaires et leur renvoient une projection grandiose d'eux-mêmes $"^{4}$.

D'évidence, les mesures de déradicalisation (ou plutôt de désengagement) doivent opérer essentiellement dans les champs sociologique, éducationnel, idéologique et psychosociologique. Aussi, nous ne traiterons pas des mesures coercitives qui opèrent dans le cadre policier et militaire, quand bien même les porosités existent comme dans le cas de certains militaires radicalisés 5 . 


\section{Le milieu carcéral}

Sachant que le milieu carcéral peut être aussi un vivier salafiste et de recrutement ${ }^{6}$, des " usines à terroristes " ou des incubateurs d'extrémismes ${ }^{7}$, un rapport de 2018 des Nations Unies sur les droits de l'Homme et les libertés fondamentales indiquait qu'il était préoccupant qu'aucun programme systématique de déradicalisation ou de désengagement n'existe en Belgique.

Pour d'aucuns, l'enfermement crée de la haine, de la colère, de l'endurcissement et du ressentiment auxquels le discours radical fait écho. La prison, incubateur de radicalisme sans nécessairement passer par le Web (ex : Mohamed Merah, Mehdi Nemmouche, Amedy Coulybably, Chérif sociales, professionnelles, émotionnelles ou intellectuelles de ceux qui y sont confinés " (éditorial de Béatrice Delvaux), le détenu cherchant à travers le radicalisme en prison un sentiment d'appartenance, un élément de fierté, un point de repère structurant et donnant sens, une garantie de protection, un certificat de détermination virile (étape dans " la carrière de djihadiste "), un appui à la résistance individuelle ou collective contre l'institution ${ }^{8}$, avec le constat final que l'on ne trouve pas à chaque fois une ferme conversion religieuse.

Selon le rapport annuel de la Sûreté de l'État 2017-2018, " de nombreux prisonniers à la recherche d'un soutien mental et psychologique dans le milieu carcéral clos et isolé, y découvrent ou redécouvrent l'Islam radical comme moyen de se réconcilier avec leur passé, de forcer le respect et d'inspirer l'autorité auprès de leurs codétenus ou comme justification pseudo-religieuse à

leur comportement criminel ". Reste que la prison serait loin d'être le premier lieu de radicalisation mais elle pourrait contribuer à l'accélération de ce processus ${ }^{9}$.

Certains auteurs estiment que ce sont les expériences pré- et post-carcérales qui doivent être considérées comme les facteurs-clés dans l'adoption d'une idéologie radicale ${ }^{10}$. Il y a bien également une frontière des plus ténue entre la délinquance et le terrorisme, le grand banditisme $^{11}$ et le trafic d'armes. Le processus d'embrigadement radical serait comparable à l'enrôlement de recrues dans le milieu mafieux et le grand banditisme. Reste que selon l'Institut flamand pour la paix, avec les bonnes connexions, les terroristes de moindre envergure sont aussi capables d'acquérir des armes " lourdes ${ }^{12}$.

Les auteurs des attentats commis en France et en Belgique en 2015 et 2016 proviendraient presque tous du monde du gangstérisme. La grande majorité de ces terroristes seraient originellement et pour l'essentiel des criminels et des délinquants de droit commun. Cette hybridation est une imbrication entre la recherche du pouvoir par destruction et celle du profit criminel (idéologie versus profit ; violence politique indiscriminé versus violence criminelle discrète).

L'idéologie salafiste peut être un masque camouflant des motivations prédatrices (violence, argent, pouvoir). Il serait plutôt question d'une " islamisation-salafisation de la criminalité de droit commun ${ }^{13}$. Il s'agirait " d'islamistes passant aisément à l'acte criminel en raison même de leur habitus de déviance de violence ". Selon Saint Victor, l'adoption du discours salafiste 
rédempteur permet de continuer en toute impunité les pratiques prédatrices en bonne conscience ; sorte de recréation au centuple de la délinquance des banlieues.

En 2018, sur les 450 détenus radicalisés, 132 ont reçu une forme d'accompagnement et 68 ont entamé un parcours individuel de déradicalisation : 33 en Flandres et 35 en Belgique francophone. Mais sur les 450 détenus radicalisés, 237 figurent sur une liste plus dangereuse, " la cellule " extrémisme " créé en 2015 par l'administration pénitentiaire. Relevons que 21 détenus parmi eux sont logés dans des ailes séparées dites " De-Radex " (prisons de Hasselt et Ittre) où ils sont isolés afin d'éviter les influences par propagation de la parole radicale. Parmi les 21, douze d'entre eux suivent un parcours de déradicalisation.

Selon un rapport de la Sûreté de l'État, en 2017, 76 personnes radicalisées ou liées au terrorisme ont été libérés de prison après avoir purgé leur peine. Selon la Sûreté, en septembre 2018, il y avait 130 détenus condamnés pour terrorisme ou en détention préventive dans le cadre d'un dossier de terrorisme. La même année, 28 détenus ont pu retrouver leur liberté. Parmi ces vingthuit individus, 12 étaient de combattant revenus de Syrie ou souhaitant s'y rendre, 10 délinquants radicalisés en prison et 6 condamnés pour leurs liens avec une organisation terroriste. Selon le ministre de la Justice, Koen Geens, depuis le début de la guerre en Syrie jusqu'à fin 2018, 182 terroristes, complices de terroristes, prédicateurs de haine et personnes radicalisées qui avaient été condamnées, ont été libérés.

Concernant les " returnees ${ }^{14}$, les individus majeurs sont auditionnés par un juge d'instruction (degré d'implication au terrorisme et aux exécutions) avant de passer par la case prison (mandat d'arrêt, détention préventive avant jugement) et tous les mineurs et les femmes revenant de la zone irako-syrienne - après quelques divergences politiques en matière d'obligation et de gestion des retours - font l'objet d'un suivi par le parquet et par les agences communautaires d'aide à la jeunesse.

Selon la Sûreté de l'État, dans son rapport 2017-2018, jusqu'en 2015, l'échange d'informations entre l'administration pénitentiaire et la VSSE s'effectuait par l'intermédiaire d'une personne de contact à la Sûreté. À la mi-2015, il fut décidé d'instaurer une "Cellule prisons " spécifique (de 2 personnels en 2015 à 12 en 2018). Cette cellule " collabore de manière intensive avec la " Cellule extrémisme " (Celex) chargée de cette problématique au sein de l'administration pénitentiaire. En plus de cette collaboration, un " groupe de travail prisons " a vu le jour il y a environ deux ans. Il s'agit d'un organe de concertation permanent présidé par la Sûreté de l'Etat et dont la mission est d'examiner des dossiers et d'échanger des informations avec l'administration pénitentiaire, l'OCAM (Organe de Coordination pour l'Analyse de la Menace) et les services centraux de la Police fédérale en charge du terrorisme (DJSOC/TERRO).

"Cet organe s'inscrit dans le cadre du Plan d'action radicalisme fédéral (Plan R). Par ailleurs, un " groupe stratégique prisons " réunit trois fois l'an de nombreux partenaires. Aux côtés de la Sûreté de l'Etat, nous y trouvons les services centraux de la Police fédérale en charge du terrorisme (DJSOC/TERRO), les établissements pénitentiaires, l’OCAM, la Direction générale du 
Centre de crise, le service de renseignement militaire (SGRS), le service public fédéral Affaires étrangères, l'Office des Étrangers, les maisons de justice (des communautés française et flamande), le secrétariat technique et administratif du Ministre de la Justice. Ces partenaires évaluent le flux d'information entre les différents services, examinent les nouvelles tendances ainsi que les approches stratégiques y relatives. En outre, afin d'optimiser les flux d'informations les différents services ont recours à la base de données communes (BDC) ".

\section{Propositions/améliorations/ décisions $^{15}$}

**Création début 2017 en Fédération Wallonie-Bruxelles et sur base volontaire pour les détenus d'un accompagnement personnalisé et confidentiel du Centre d'Aide et de Prise en charge de toutes personnes concernées par les Extrémismes et Radicalismes Violents (Caprev) pour préparer à la réinsertion sociale, via le désengagement, suivi psychologique visant à faire renoncer à toute action violente ${ }^{16}$.

**Nécessité de meilleurs échanges entre les prisons et la Sûreté de l'État (Cf. Commission d'enquête parlementaire + réactions au terrorisme à Liège du 28 mai 2018).

** « Les terroristes en détention ou les criminels fortement radicalisés ne devraient pas être libérés tant qu'il existe une menace terroriste " (Position de Bart De Wever, interview, VRT, 1 juin 2018).

**Proposition électorale de la NV-A de créer des prisons belges à Rabat et à Agadir pour y transférer les détenus marocains condamnés en Belgique (droit commun et radicalisés).
${ }^{* *}$ Equiper les suspects de radicalisme non condamnés mais soupçonnés d'appartenance à des milieux radicaux et aux radicaux libérés de prison afın de devoir porter un bracelet électronique (Proposition de Denis Ducarme, alors Chef de Groupe MR à la Chambre, 15 janvier 2015).

**Isolement de détenus prosélytes et prédicateurs de haine et monitoring ${ }^{17}$ de détenus susceptibles de se radicaliser (Décision du ministre de la Justice, Koen Geens (CD\&tV) en 2015).

**Souhait des directeurs régionaux des prisons pour disposer, comme en France, de services secrets pour les prisons (2017). Refus du ministre de la Justice privilégiant l'agent d'information dans chaque prison (Information officer) et les services de l'OCAM.

**Projet spécifique en Flandres destiné aux détenus en liberté conditionnelle via le financement de deux Imams " pour amener à de meilleurs sentiments " lesdits individus (Décision du ministre du Bien Etre, Jo Vandeurzen, CD\&tV, juin 2018).

**Pédagogisme (ex : Présentation aux détenus de Ittre de la pièce de théâtre " Lettres à Nour " de Rachid Benzine, juin 2018) ${ }^{18}$.

**Augmenter la formation réalisée du personnel pénitentiaire en matière d'identification et de surveillance de radicalisés ${ }^{19}$ (Ittre, Hasselt, Gand, Bruges, St-Gilles, Andenne, Marche-en-Famenne et Lantin). Début mars 2019, environ 456 agents pénitentiaires avaient suivi une formation sur la radicalisation, tandis que 70 autres, employés au sein de sections spécialisées, 
ont assisté à des cours plus approfondis. Existe également un module d'apprentissage en ligne sur tous les extrémismes, proposé à l'ensemble des agents et financé par des fonds européens.

\section{La Dé-radicalisation en milieu local}

Dans la lutte contre la radicalisation violente, les aspects locaux sont primordiaux. La Cour des comptes européenne ne s'est pas trompée en examinant les mesures prises par l'UE pour aider les États membres. Mais d'avertir que " la Commission ne dispose pas d'une vue exhaustive des actions financées par l'Union et les fonds de l'UE utilisés ne sont assortis d'aucun indicateur ou objectif chiffré permettant de mesurer la réussite en matière de lutte contre la radicalisation ". Aussi, "les auditeurs ont constaté que le réseau de sensibilisation à la radicalisation, qui met en relation des psychologues, des enseignants, des assistants sociaux, des policiers, des agents pénitentiaires et des agents de probation de toute l'Europe qui travaillent directement auprès des individus vulnérables à la radicalisation, n'a pas été pleinement exploité.

Les réalisations obtenues grâce à ce réseau étaient souvent mesurées en volume d'activité (par exemple le nombre de réunions organisées ou de documents établis) plutôt que sous l'angle de l'efficacité (par exemple les connaissances acquises ou leur impact sur le travail des participants)".

Dans le cadre du " plan d'action radicalisme ", lancé en juin 2016, quatorze LTF à l'échelle du Royaume de Belgique ont été mises sur pied. Ces plateformes locales sont destinées aux services de police et de renseignement (approche réactive et répressive). Elles sont installées dans des zones stratégiques et doivent garantir le suivi d'individus radicalisés. Elles ont pour mission d'accompagner les djihadistes de retour en Belgique, mais aussi d'entretenir des contacts avec les autorités locales ${ }^{20}$. Précisons que ces 14 tasks forces locales impliquent une concertation mensuelle avec les services de sécurité (police locale, police fédérale, parquet, sûreté de l'État et OCAD).

En outre, sur proposition du ministre de la Sécurité et de l'Intérieur Jan Jambon, le Conseil des Ministres du 27 avril 2018 a approuvé un avant-projet de loi portant création de cellules de sécurité intégrales locales (CSIL) ${ }^{21}$ en matière de radicalisme, d'extrémisme et de terrorisme (approche pluridimensionnelle, préventive, proactive et détection).

La CSIL est la plateforme locale permettant aux services de prévention sociale, à l'administration et à des acteurs de la société civile d'aborder des cas de radicalisation. L'organisation de la CSIL relève de la compétence du bourgmestre. Pour pouvoir en assurer le bon fonctionnement, la participation de représentants de services relevant de la compétence des entités fédérées serait toutefois également souhaitable. Il appartiendrait aux entités fédérées - si ces dernières l'estiment nécessaire - de prendre des initiatives permettant de renforcer le fonctionnement des CSIL.

L'avant-projet de loi, qui s'inscrit dans le cadre des recommandations de la commission d'enquête parlementaire $d u$ 22 mars, vise à créer des cellules de sécurité intégrales locales (CSIL) pour chaque commune et défınit le cadre minimal pour 
le fonctionnement de ces cellules. Relevons que ladite cellule se réunit à la demande de l'un des membres et/ou lorsqu'un cas individuel doit être discuté, afin de ne pas augmenter inutilement la charge de travail des communes. Ainsi, s'il n'y a pas d'éléments de radicalisation ou de menace terroriste imminente, la CSIL peut être maintenue en " mode veille ${ }^{22}$.

Le 16 juillet 2018, un projet de $\operatorname{loi}^{23}$ de la Chambre des Représentants portait création de cellules de sécurité intégrales locales en matière de radicalisme, d'extrémisme et de terrorisme était adopté par 119 voix (et 20 abstentions). L'idée première est que chaque commune soit ainsi couverte par une taskforce locale et une cellule de sécurité intégrale locale (CSIL) car les radicaux peuvent s'établir partout sur le territoire.

Plus de la moitié des 589 communes belges ${ }^{24}$ avaient déjà créé leur propre CSIL ou font partie d'une telle cellule : celle-ci peut en effet être créée par une commune/ville ou être organisée au niveau supralocal. Sa composition et son fonctionnement varie encore fortement. Afin d'uniformiser les cellules, une loi fédérale du 30 juillet 2018 prévoit, outre l'obligation de créer une CSIL au niveau local ou supralocal, également l'organisation de celle-ci sous la direction du bourgmestre ${ }^{25}$ et la participation à la carte de certaines fonctions (telles que les fonctionnaires de déradicalisation, enseignants, directeurs d'écoles, responsables logements sociaux, CPAS, travailleurs du service prévention,...) et la présence de l'Information Officer de la police locale (qui sert de relais avec la Taskforce locale).

Malgré la prescription légale (mais sans pouvoir de contrainte du ministère), au $1^{\text {er }}$ janvier 2019, 138 communes sont sans cellule antiterroriste (31 en Flandre et 107 en Wallonie) alors que 347 communes en sont dotées (236 cellules par association).

Relevons qu'à Verviers, existe le Service d'Accompagnement des Familles et de l'Entourage en matière de radicalisme (SAFER) ${ }^{26}$ où un islamologue et une criminologue aident une dizaine de familles à se tourner vers un islam modéré en les orientant vers des imams reconnus et à parfaire leur esprit critique.

Le principe est d'écouter, aider et accompagner toute personne concernée directement ou indirectement par le phénomène $\mathrm{du}$ radicalisme. L'action de ce service ${ }^{27}$ permettrait de récolter des informations auprès des écoles, des administrations, des entreprises et des citoyens qui constateraient des comportements suspects (1) ; d'accompagner les familles qui se trouvent dans une situation de grand désarroi face à la radicalisation d'un membre de la famille (2) et d'accompagner et d'orienter les jeunes vers des imams modérés qui disposent d'une autorité morale pour les mener vers la déradicalisation (3).

\section{Propositions/améliorations/ décisions ${ }^{28}$}

**Proposition de loi du gouvernement Michel adoptée en mai 2017 de contraindre les assistants sociaux des CPAS de transmettre " sans délai ou sans perdre de temps " les renseignements que leur demandera un procureur du Roi ${ }^{29}$ (communication passive) et de lui déclarer de manière proactive (communication active) et "sur-le-champ " tout indice sérieux d'existence d'une infraction terroriste (le " secret professionnel 
partagé "). La Cour constitutionnelle a annulé le 15 mars 2019 lesdites dispositions concernant la dénonciation proactive des suspects par les assistants sociaux mais les personnels sociaux devront toujours répondre aux questions du Procureur du Roi et délivrer des informations couvertes par le secret professionnel, dans le cadre, bien précis, d'enquêtes sur des faits de terrorisme.

**Création en janvier 2017 du Centre d'Aide et de Prise en charge de toutes Personnes concernées par les Extrémismes et Radicalismes Violents (CAPREV) pour la Fédération Wallonie-Bruxelles avait pour objectif de recevoir des coups de fil sur son numéro vert (080011172) concernant une aide à apporter à des jeunes en voie de radicalisation. Depuis son lancement en janvier 2017, le CAPREV a reçu plus de 1.000 appels téléphoniques. Près de 150 dossiers ont été ouverts et 88 font encore l'objet d'un suivi actif en mars 2019.

**Mise sur pied par l'OCAM d'un "Centre de connaissance " ayant pour but de dresser la carte de l'offre de déradicalisation en Belgique, faciliter la coordination et partager les avis. Il s'agit d'aboutir à des standards minimums communs car les programmes se sont multipliés et il existerait des " boîtes " vides et des initiatives purement commerciales.

** En décembre 2017, dans le cadre du Plan de prévention du radicalisme et à l'initiative de la Ministre des Pouvoirs locaux, Valérie De Bue, le Gouvernement wallon a donné son accord pour lancer un deuxième appel à projets intitulé « Prévention de la radicalisation violente " s'adressant aux communes disposant d'un plan de cohésion sociale.
Suite au premier appel à projets 2017 intitulé "Amélioration du vivre ensemble et prévention du radicalisme ", Valérie De Bue, en concertation avec le Ministre-Président, a tout d'abord souhaité évaluer les projets pour étudier le lancement d'un deuxième appel. Sur base du rapport d'évaluation de l'administration, la Ministre a proposé une nouvelle mouture d'appel à projets en les axant davantage :

- Sur la prévention de la radicalisation violente ;

- Sur la mise en place de projets concrets ayant dépassé le stade de la réflexion ;

- Sur la formation des acteurs de première ligne, tant au sein des pouvoirs publics que dans les associations culturelles, sportives ou à dimension sociale (représentants de la/des commune(s), du/des CPAS, de la Police ainsi que tout partenaire jugé pertinent en lien direct avec la thématique de prévention contre la radicalisation violente (imams, maisons de jeunes, secteur d'aide à la jeunesse, écoles, centres régionaux d'intégration,...).

À ce titre, un des prérequis pour répondre à cet appel à projets est l'existence d'une plateforme de concertation au niveau communal ${ }^{30}$ réunissant tous ces acteurs en contact direct avec un large éventail de la population. Il s'agira pour les communes de présenter des projets concrets qui ont dépassé le stade de la réflexion et qui sont en adéquation avec les besoins identifiés dans un diagnostic en matière de prévention de la radicalisation violente. Sur les 18 candidatures reçues au ministère wallon des pouvoirs locaux, 4 communes ont été retenues en 2018 : Aiseau-Presles, Farciennes, Dison et Visé. 
***eréation début 2019, par le gouvernement wallon, d'un plan de lutte contre le radicalisme violent (enveloppe de 4,4 millions d'euros) en dix mesures concrètes :

1. Création d'un Centre régional d'expertise en matière de prévention du radicalisme violent Ce centre aura pour vocation d'être un véritable centre d'excellence rassemblant tous les professionnels de la Wallonie en la matière, permettant dès lors la cohésion des différents partenaires.

2. Appui renforcé aux Cellules de sécurité intégrale locales en matière de radicalisme, d'extrémisme et de terrorisme Depuis juillet 2018, les bourgmestres sont tenus de créer une cellule de sécurité intégrale locale en matière de radicalisme, d'extrémisme et de terrorisme (CSIL-R). Celle-ci a pour but de prévenir des infractions terroristes. 74 communes disposent déjà d'une telle cellule. La Wallonie accordera des moyens financiers supplémentaires aux villes et communes wallonnes, via un appel à candidatures, pour engager un coordinateur chargé de superviser les travaux et les réunions de la cellule.

3. Mise en place d'une mobile team régionale Une mobile team sera mise en place au sein du SPW Intérieur et Action sociale afin d'améliorer la couverture du territoire wallon en matière de services de formation à la détection du radicalisme. Les communes pourront faire appel à cette équipe mobile pour leur permettre de former leur personnel à la détection du radicalisme.

4. Administration : prévenir la criminalité liée au radicalisme

Une étude doit être réalisée en vue de mettre en place une approche administrative de prévention de certains risques au regard des valeurs démocratiques de la société wallonne (activation de dispositif des sanctions administratives, agrément ou retrait d'agrément de certains établissements, etc.).

5. Inventaire des lieux sensibles du territoire wallon

Par site sensible, il faut entendre des infrastructures qui nécessitent des mesures de sécurité particulière pour prévenir toute infiltration par une personne radicalisée et souhaitant passer à l'acte sous la forme d'une attaque terroriste.

6. Formation spécifıque à destination des fonctionnaires régionaux

Sensibiliser et informer des agents de terrain en lien direct avec la population à certains phénomènes criminels, comme le radicalisme violent est essentiel. Un nouveau programme de formation spécifique sera également mis sur pied. Il s'adressera en priorité aux agents du SPW, aux organismes publics régionaux et au Réseau radicalisme, le public cible pouvant être étendu par la suite.

7. Plan de sécurité interne au SPW

L'ensemble des plans internes d'urgence des bâtiments du SPW seront étendus aux aspects de sécurité et prévoiront donc également des réponses adaptées en cas de vols, d'intrusions, d'actes de terrorisme, de vandalisme, etc.

8. Renforcement des dispositifs visant l'intégration et le "vivre ensemble "

Cette mesure vise à assurer la diffusion des outils d'information et des bonnes pratiques relatifs à la lutte contre le radicalisme violent auprès des organismes et asbl actifs 
dans le domaine du vivre ensemble, de la cohésion sociale, de la lutte contre les discriminations.

9. Décryptage de l'information pour développer l'esprit critique sur les réseaux sociaux Il s'agit de favoriser/encourager des projets de veille sur les réseaux sociaux et de déconstruction des discours radicaux sous toutes ses formes : discours de haine, théories du complot, appels à la violence, propagande, etc.

10. Formation professionnelle à destination des personnes détenues Afin de réduire le risque de récidive, notamment dans le chef des personnes radicalisées qui séjournent dans une prison wallonne, et pour lutter contre l'endoctrinement religieux en milieu carcéral, il est nécessaire de poursuivre et accentuer les efforts de formation des détenus pour faciliter leur sortie de prison et leur réinsertion.

\section{La Dé-radicalisation en milieux éducationnels}

Le domaine de l'éducation est l'élément premier et prioritaire afin de pouvoir réduire le radicalisme violent, d'autant que les djihadistes avaient organisé un enseignement spécifique pour les jeunes ${ }^{31}$ et les familles arrivant dans la zone de Daesh. Toute la difficulté des politiques de désengagement par l'éducation sont d'autant plus complexes que certains jeunes sont en voie de réinsertion tandis que d'autres peuvent manipuler ou être en revirement ${ }^{32}$. La radicalisation est " un problème d'ordre pédagogique " imposant un soutien familial (sachant aussi que la famille peut être le lieu de transmission du djihadisme) autant qu'une démarche évitant trop de suspicion, quand bien même il n'y a aucune manière de prévoir ou d'anticiper le fait qu'un individu devienne ou non un terroriste.

Et pour complexifier le tout, il existe des écoles privées, des écoles coraniques, l'enseignement à domicile ${ }^{33}$ et l'influence " éducative " des imams organisant les " casemates identitaires " (Expression d'Hassan Bousetta, ULg).

L'objectif premier pourrait être le développement d'un dispositif d'instruction qui dispense une culture ouverte à l'universel et qui émancipe la capacité de raisonner de manière autonome en fournissant des références non partisanes.

Parallèlement et au niveau macro, la lutte idéologique passe par le contrôle complexe de l'internet, des plateformes de diffusion (YouTube et Dailymotion) et des réseaux sociaux (MSN, Twitter, Facebook " anonymisé ", Telegram et des support Microsoft et Apple) dès lors que la sensibilité des jeunes aux processus de propagande d'internet est bel et bien démontrée ${ }^{34}$ et que cela permet de booster le passage à l'acte (cf. Supra).

Il s'agit alors, en réponse, dans les différents espaces d'engagement (locaux, nationaux, multinationaux, géants d'internet) de favoriser l'encouragement aux messages anti-radicaux ou d'organiser la vérification des faits et des fausses nouvelles via, par exemple, une collaboration d'organes de presse traditionnels. Il s'agit aussi de fermer des milliers de comptes, de supprimer plus rapidement des contenus, organiser des algorithmes de redirection, de travailler également "sur la réceptivité de certains individus au Web $"^{35}$. En effet, le contact 
personnalisé (virtuel ou physique) via le Web est assez systématique.

Toute la difficulté étant la résilience des comptes qui se recréent et donc l'illusion de pouvoir interrompre totalement les réseaux aux messages djihadistes, quand bien même les bombardements ont impacté l'appareil, en partie centralisé, de propagande ${ }^{36}$ par rétrécissement territorial, y compris par neutralisation des responsables des médias de Daesh. L'épuration complète du net étant illusoire pour des motifs techniques, juridiques, politiques et financières (Marthoz), les dimensions de contre-propagande, du contre-narratif et d'éducation aux médias deviennent prioritaires.

Parallèlement à l'ensemble de ses politiques de soutien au vivre ensemble et à la citoyenneté responsable (par la culture, l'éducation, le sport), la Fédération Wallonie-Bruxelles poursuit sa politique de prévention des extrémismes menant à la violence. Aussi, dans le cadre du renforcement de ces initiatives et suite à l'avis favorable du comité d'experts, le Centre de Ressources et d'Appui pour la prévention des extrémismes et des radicalismes violents (CREA) de la Fédération Wallonie-Bruxelles, réitère sa proposition de deux outils afın d'appréhender le phénomène du radicalisme au sein des écoles.

Le premier outil s'intitule " Le terrorisme en question ". Sur base de ses outils pédagogiques développés sur le terrorisme et sur la radicalisation des jeunes belges engagés dans le djihadisme, la Coordination Nationale d'Actions pour la Paix et la Démocratie (CNAPD) propose des animations centrées sur les enjeux politiques qui sous-tendent ces questions. Prévue pour une classe, la durée de l'animation n'excèdera pas une demi-journée. Le public visé par cette activité est le groupe-classe de $4^{\mathrm{e}}, 5^{\mathrm{e}}$ et $6^{\text {e }}$ années du secondaire. Les objectifs recherchés : prendre du recul ; Identifier des représentations et les mettre en question ; Accompagner la volonté de comprendre : rechercher, identifier et interroger un faisceau de causes ; Proposer une information critique, des références, des pistes plurielles de lecture et d'interprétation ; Promouvoir l'exercice de la citoyenneté et de l'action non-violente.

Le second outil s'intitule " Rien à faire, rien à perdre " (RAFRAP). Il s'agit d'animations avec support pédagogique. Les capsules sont accompagnées d'un dossier pédagogique rédigé par Anne-Marie Impe, journaliste indépendante et formatrice, à la demande du Centre de Ressources et d'Appui du Réseau de prise en charge des extrémismes et des radicalismes violents de la Fédération Wallonie-Bruxelles.

Lesdites capsules reprennent le témoignage de jeunes Belges qui ont eu pour projet de rejoindre des mouvements islamistes radicaux en Syrie et en Irak. Le portrait de proches et de membres de familles concernées (sœur, mère, père, enseignante, etc.) apporte également un éclairage sur les conséquences de ces engagements. À partir de leur récit, l'ASBL S.A.V.E. Belgium propose une animation de deux heures de cours. Le public visé par cette activité est celui des classes de $1^{\mathrm{er}}, 2^{\mathrm{e}}, 3^{\mathrm{e}}, 4^{\mathrm{e}}, 5^{\mathrm{e}}$ et $6^{\mathrm{e}}$ années du secondaire. Les objectifs recherchés sont : intégrer les notions de radicalisme violent ; mieux comprendre les techniques de prosélytisme et de recrutement ; proposer une information critique, des références, des pistes plurielles de lecture et d'interprétation. 
"Rien à faire, rien à perdre " (RAFRAP) est un projet ${ }^{37}$ de recueil de récits de vie, présentés sous la forme de capsules vidéo de 4 à 6 minutes. Ce projet a été conçu et mis en œuvre par Isabelle Seret, formatrice et intervenante en récit de vie et sociologie clinique.

Relevons qu'entre janvier 2015 et juin 2016, le service des équipes mobiles de la Fédération Wallonie-Bruxelles avait reçu de la part des chefs d'établissements scolaires, 77 demandes d'intervention liées à la thématique du radicalisme menant à la violence (dont 60 concernaient des inquiétudes vis-à-vis d'un élève, dont 12 imposant un relais à la police locale).

\section{Propositions/améliorations/ décisions $^{38}$}

*Débat sur l'instauration d'un Service citoyen civil en Belgique dont le dispositif pourrait influer aussi positivement sur la déradicalisation ;

*Dépôt de propositions au parti libéral francophone (MR, janvier 2016) autour des signes convictionnels et la neutralité, dont « la mise à l'index des signes convictionnels dans les écoles du réseau officiel ", " évaluation de l'opposition à certains cours ou activités scolaires " et " formation des professeurs à l'interculturalité ${ }^{39}$ et à la gestion des différences culturelles entre élèves ".

*Relance des débats déjà anciens sur la place de la laïcité en Belgique face au radicalisme et aux dogmatismes religieux.

*Organiser concrètement des échanges et activités communes entre jeunes d'espaces culturels différents (Andrea Rea).
*Lancement par la Fédération WallonieBruxelles $^{40}$ en septembre 2017 des cours d'éducation à la philosophie et à la citoyenneté (CPC) dans l'enseignement fondamental ${ }^{41}$ puis secondaire officiel $\left(1^{\mathrm{er}}\right.$ et $2^{\mathrm{e}} / 3^{\mathrm{e}}$ degré) et débats sur la place des cours convictionnels dans le cursus scolaire, le contenu des cours et des unités d'acquis d'apprentissage, de même que l'apprentissage du " fait religieux " dans le CPC. L'objectif est le "vivre ensemble ». Dans l'introduction du décret, nous pouvons lire que l'EPC « vise une compréhension pluraliste et critique des enjeux de la citoyenneté ; c'est à ce titre que la démarche philosophique doit guider l'ensemble de ses apprentissages. Comme réflexivité critique, la philosophie apprend en effet à comprendre la pluralité des logiques, des normes d'action et des valeurs dont l'humanité comme irréductiblement plurielle est porteuse, et à construire des outils conceptuels et des attitudes pour juger et décider en autonomie, en étant capable de mettre à distance ses propres évidences, ses propres catégories et façons de penser. En prenant en compte les apports des autres disciplines, en particulier des sciences humaines et sociales et de l'histoire des religions et de la laïcité, la réflexion philosophique contribue à la formation de citoyens ouverts et autonomes".

Pour ne pas alourdir leur horaire, il fut décidé que les cours de religion et de morale passeraient de deux heures par semaine à une heure par semaine. Il fut décidé que les élèves qui ne souhaitaient ne plus suivre de cours de morale ou de religion puissent remplacer ce cours par une heure supplémentaire d'éducation à la philosophie à la citoyenneté. Selon l'Observatoire des religions et de la laïcité, 12,2\% des parents d'élèves de primaire et 15,3\% des parents 
d'élèves de secondaire ont opté pour une seconde heure d'éducation à la philosophie et à la citoyenneté (EPC) pour l'année 20182019. L'année précédente, ces pourcentages s'établissaient à 10,9 au niveau primaire et à $12,1 \%$ en secondaire. Depuis qu'il est possible d'opter pour deux heures de cours d'éducation à la philosophie et à la citoyenneté, les cours de religion catholique et de morale non confessionnelle connaissent une baisse significative de leur fréquentation, tandis que les cours de religion islamique, protestante et orthodoxe continuent de voir leur fréquentation augmenter, note l'Observatoire. C'est le cours de morale qui serait le plus fortement touché par cette diminution.

Selon cette étude réalisée par Caroline troduction du CPC n'a visiblement pas résolu une série de problèmes posés ou rencontrés par les cours de religion et de morale, tels la séparation des élèves sur une base convictionnelle ou les lacunes dans la formation des enseignants de religion et dans l'élaboration du programme de leurs cours. Des deux côtés de la frontière linguistique, il existe des demandes pour de nouvelles réformes de l'organisation des cours philosophiques. Si en Flandre, des organisations issues de la société civile plaident de longue date pour l'introduction d'un cours semblable au CPC du côté francophone, en Communauté française, des voix soutiennent la généralisation des deux heures de CPC à tous les élèves, les cours de religion et de morale étant soit rendus facultatifs soit franchement supprimés ".

*Organisation par l'université de Liège (campus Liège et Arlon) d'un certificat en Didactique de la Philosophie et de la

Citoyenneté à partir de l'année scolaire 2018-2019 au profit des personnels enseignant dans un établissement secondaire supérieur ( 3 à $6^{\mathrm{e}}$ ) de religion et de morale, du réseau officiel ou libre non confessionnel, en province de Liège et de Luxembourg.

\section{Conclusion provisoire}

Le processus de désendoctrinement, désembrigadement, désengagement, " désistance " et de déradicalisation est infıniment complexe et beaucoup de programmes ont échoué ; l'idéologie totalitaire pouvant contourner les procédés de contre-radicalisation. Les explications plurielles $d u$ processus de motivation et d'engagement vers la radicalisation violente semblent bien saisies mais elles imposent encore modestie et prudence dans la mesure où l'on constate des lacunes en matière d'études quantitatives (selon Michaël Dantinne, criminologue à l'ULg, CETR).

Il manque encore une échelle de mesure véritable, l'accès libre aux données étatiques alors que les programmes de déradicalisation sont encore assez récents ${ }^{42}$. En Belgique, nous sommes encore dans les expérimentations ou les doutes sur l'efficacité des programmes de prévention (selon Vincent Seron, criminologue à l'ULg, CETR). Il s'agit aussi de tenter de maîtriser la grille individuelle d'interprétation de la réalité des radicalisés violents et ceux en voie de l'être potentiellement.

Il nous faut accepter l'incertitude sur les postures radicales et leurs justificatifs parfois " leurrants ". Déceler les signaux faibles et intervenir en amont n'est pas une science exacte. 
De plus, il nous faut aussi sortir des débats polémiques et polarisant autour des thèses de Gilles Kepel et Olivier Roy tels qu'ils existent en France : Islamisation de la radicalité, radicalisation de l'islam ; sachant qu'il y a bien complémentarité de ces deux thèses sur fond de complexité à lire le phénomène.
Le radicalisme violent impose des politiques de prévention : la prévention cognitive par l'éducation, le vivre ensemble et la lutte contre l'idéologie violente ; la prévention d'évitement de la délinquance et enfın la prévention par la résilience, la " réhumanisation " et un projet de vie social.

\section{Notes}

* Attaché à l'Institut royal supérieur de défense (IRSD). Professeur à l'Université de Liège. Membre du CETR.

1. Vision stratégique pour la défense, version illustrée sur http://www.vandeput.fgov.be/ sites/default/files/articles/Strategic-Vision-Belgian-Defense-FR.pdf

2. Pour une analyse du concept de prévention et ses différentes approches interprétatives, cf. Vincent SERON, Prévention de la radicalisation : aperçu des différents modèles utilisés à l'échelle internationale, CETR, ULg, 27 avril 2018 (document non accessible).

3. Dounia Bouzar parle même de " désistement " pour ceux qui ont fait le deuil à la fois de l'utilisation de la violence et de l'idéologie qui la sous-tend (Dounia BOUZAR, Étapes du processus de radicalisation et de déradicalisation, Practicies, projet de recherche, mai 2018, p. 12).

4. Bilel AININE, Xavier CRETTIEZ, Thomas LINDEMANN, Romain SEZE, Saisir les mécanismes de la radicalisation violente. Pour une analyse processuelle et biographique des engagements violents djihadistes et nationalistes, Rapport de recherche pour la mission de recherche Droit et Justice, Paris, avril 2017, p. 10.

5. Rapport d'activités 2015, Comité Permanent R, Bruxelles, 2016, pp. 16 à 21 ; Questions et réponses parlementaires, Chambre des Représentants de Belgique, Bruxelles, 24 mars et 1 décembre 2014, 29 avril 2016, 14 juin 2017 ; Philippe MANIGART et Delphine RESTEIGNE, Sortir du rang. La gestion de la diversité à la Défense belge, Académie royale de Belgique, Bruxelles, 2013 ; Anaïs Reding et autres, La gestion de la diversité ethnique dans les armées. Le cas du Royaume-Uni, des Etats-Unis, de la Belgique et des Pays-Bas, Cahiers de l'IRSEM, $\mathrm{n}^{\circ} 17$, Paris, 2012.

6. Frank BULINGE, « La radicalisation en prison : mythe ou réalité ? Journal for Communication Studies, vol. 9, n², 2016, pp. 183-184.

7. Claire de GALEMBERT, Céline BÉRAUD \& Corinne ROSTAING, " La religion, rouage de gouvernementalité dans les prisons françaises ", Revue de droit pénal et de criminologie, Paris, 2018, pp. 283-311.

8. Cf. Rachel SARG, La foi malgré tout. Croire en prison, PUF, Paris, 2016 (chapitre 10) ; Philippe POISSON, De la religion en prison, Presses universitaires de Rennes, collection " science des religions ", 2016.

9. Cf. Vincent SERON, Prévenir et gérer la radicalisation (violente) en milieu carcéral : éléments de compréhension et pistes de réflexion, CETR, Module " Prévention et répression ", ULg, 2018 (document non accessible).

10. Ibid.

11. Rapport d'activité 2017-2018, Sûreté de l'Etat, Bruxelles, 2018, p. 17.

12. Cf. aussi Jean-Claude ANTOINE, Au cœur des trafics d'armes. Des Balkans aux banlieues, éd. Vendémiaire, Paris, 2012 ; Communiqué de presse du Conseil de l'UE, 12 décembre 2018 et 14 février 2019 ; Jean-François GAYRAUD, Théorie des hybrides. Terrorisme et crime organisé, CNRS Editions, Paris, 2017 ; Alain BAUER, Crime et terrorisme : sous-traitants, associés ou hybrides ? CSFRS, Paris, 5 mars 2018 ; Agence Belga, 18 avril 2018. 


\section{André DUMOULIN}

13. Mathieu FRUSTIÉ, "Les liens entre le terrorisme et la criminalité organisée ", Défense \& Sécurité internationale, hors-série n²4, Areion, Paris, avril-mai 2016.

14. Selon le directeur de l'OCAM dans une interview à Het Laatste Niews du 15 janvier 2019, sur les 100 combattants étrangers qui sont revenus en Belgique depuis la Syrie, un quart présenterait encore un risque terroriste sérieux. Selon la même source, 422 foreign terroriste fighters qui ont quitté la Belgique depuis le début du conflit en Syrie, 130 sont déjà rentrés et 142 seraient officiellement décédés. Théoriquement, resteraient 150 Belges mais dont une grande partie aurait été tués ces derniers mois par les frappes aériennes, les forces kurdes et Daesh en représailles à des tentatives de désertion ou de trahison.

15. Rapport de la Sûreté de l'Etat 2017-2018, Bruxelles, 16 octobre 2018 ; Het Laatste Niews, 15 janvier 2019 ; Vincent Seron, Prévenir et gérer la radicalisation (violente) en milieu carcéral : éléments de compréhension et pistes de réflexion, CETR, Ulg, 27 avril 2018 (non accessible), Agence Belga, 2018 et 2019.

16. Discussion sur le sens de la spiritualité et les limites entre la religion et la violence ; ouverture au dialogue sur la religion. Pas de contre-discours car cela peut renforcer certains radicalisés à la pensée binaire et qui ne sont pas prêt à entende d'autres discours (Lorraine Kihl, "Quels moyens contre le radicalisme en prison ? ", Le Soir, 30 mai 2018).

17. A savoir un rapport chaque vendredi rédigé par le chef de quartier de la prison pour le chef de garde sur le comportement de ses prisonniers. Ce dernier transmet le rapport à la Cellule extrémisme dans les prisons (CellEx) qui récolte tout puis qui les partage par la Sûreté de l'Etat et l'OCAM.

18. Pour Rachid Madrane, ministre en charge de l'aide aux détenus dans le champ psychosocial en Fédération Wallonie-Bruxelles, " la promiscuité, la violence, le manque de perspective " dans un univers carcéral qui est, par nature, totalitaire, impose "de donner plus d'accès au sport, à la culture et à la formation " afin de favoriser la réinsertion des détenus et lutter contre la récidive estimée à 57\% en 2015. (Le Soir, 4 juin 2018). Cf. aussi l'autre pièce de théâtre de Rachid Benzine intitulée " Pour en finir avec la question musulmane " et la pièce "Djihad " d'Ismael Saidi, reconnue d'utilité publique pour les jeunes.

19. Accoutrement, comportement religieux, culte, alimentaire, humeurs, docilité, lectures, relation avec le personnel et les autres détenus (contacts, fréquences, lieux).

20. Valentine ARAM, "Comment nos voisins européens accueillent les djihadistes de retour d'Irak et de Syrie ", Le Figaro, 1 février 2018.

21. Le 21 août 2015, une circulaire avait déjà encouragé les communes à créer ce type de structure.

22. Au $1^{\text {er }}$ mars 2018, les communes les plus exposées au djihadisme sont à Bruxelles capitale (269 personnes) dont Molenbeek (72), Schaerbeek (41), Anderlecht (28), Laeken (25), Bruxelles-ville (22). En Wallonie (79 personnes) dont Liège (14), Verviers (14), Jumet (6). En Flandres (264 personnes) dont Anvers (66), Borgerhout (30) et Vilvorde (30).

23. Katja GABRIËLS, Rapport, doc 54 3209/003, Chambre des Représentants, Bruxelles, 16 juillet 2018.

24. Certaines communes restent réticentes, argumentant de leur caractère rural peu propice à la présence des radicaux violents.

25. À Verviers, la CSIL se réunit toutes les 6 semaines.

26. Cf. Corinne TORREKENS et Joël PRIVOT (dir.), Rapport de la recherche "Radicalisation " dans le cadre de la création du SAFER - Ville de Verviers. L'impact des questions de terrorisme et de radicalisation sur le vivre-ensemble à Verviers, DiverCity et Tellus, mai 2017. Certaines sources ont critiqué le coût de l'étude (40.000 euros) par rapport au résultat obtenu. 27. Synthèse des débats sur le radicalisme organisé par l'USC de Verviers le $1^{\text {er }}$ octobre 2016. 28. Katja GABRIËLS, Projet de loi portant création de cellules de sécurité intégrales locales en matière de radicalisme, d'extrémisme et de terrorisme, rapport, doc 54 3209/003, Chambre des Représentants, Bruxelles, 16 juillet 2018 ; Le Vif/L'Express, 17 février et 3 juillet 2017 ; Le Soir, 4 août 2017 ; " Questions à la Une ", RTBF, 21 mars 2018 ; https://www.wallonie. 
be/fr/actualites/plan-regional-de-lutte-contre-le-radicalisme-violent; Projet de loi visant à renforcer la lutte contre le terrorisme, Document 54, Chambre des Représentants de Belgique, Bruxelles, 22 juin 2015 ; Agence Belga, 2016 à 2019.

29. Amende à la clef si refus.

30. Les objectifs poursuivis par cette instance sont : le contact entre les services, l'échange de vue, la circulation de l'information ; des propositions de solutions ou d'explications aux communes ; permettre l'identification précoce de la radicalisation en posant un diagnostic avant l'action.

31. Il y avait trois niveaux d'études lors des expériences locales entreprises en Syrie : de 6 à 10 ans, de 11 à 15 et pour les plus de 15 ans. Par la suite, après la prise de Mossoul par les djihadistes, le bureau de l'enseignement (" diwan al-Taalim ") va produire des communiqués impliquant la mise en place d'un nouveau cursus qui exclut officiellement certaines matières ; y compris des séances de repentance et de rééducation de tout le personnel de l'Éducation nationale exerçant ou voulant exercer sur le territoire de l'État islamique. Les enseignements sont religieux, linguistiques et dogmatiques à travers le Coran ; les cours de physique, chimie et mathématiques sont associés à la maîtrise des armes et des explosifs. (Wassim NASR, "A l'école de l'Etat islamique : les "Lionceaux du Califat ", Inflexions, n³7, Ecole militaire, Paris, 2018, pp. 25-33).

32. Marion Van San (Université Erasmus de Rotterdam), à propos de son étude sur " La prévention de la radicalisation et les possibilités de déradicalisation " pour le compte du think tank Itinera Institute (Agence Belga, 13 décembre 2018).

33. Selon la Sûreté de l'État belge (rapport d'activité 2017-2018), près de 20\% de parents d'élèves inscrits dans l'enseignement à distance seraient liés à des groupes extrémistes salafistes avec par ailleurs la volonté d'isoler les filles à l'âge de la puberté.

34. Serge GARCET, Propagande, communication et radicalisation djihadiste, CETR, ULg, 2018 (non accessible).

35. Cf. à ce sujet, Martin BOUCHARD (dir.), Social Networks. Terrorism and Counter-terrorism. Radical and Connected, Routledge, New York, 2015.

36. Pierre CONESA, François-Bernard HUYGHE et Margaux CHOURAQUI, " La communication francophone de Daesh ", www.geostrategia.fr, 6 octobre 2017.

37. Ce projet est soutenu par la Commune de Schaerbeek, l'asbl S.A.V.E. Belgium, la Fondation Roi Baudouin, l'asbl BRAVVO, la Loterie Nationale, le Délégué général aux droits de l'enfant de la Fédération Wallonie-Bruxelles, le projet PRACTICIES, le GSARA, le Réseau international de sociologie clinique.

38. Décret FWB, 22 octobre 2015 ; www.news.uliege.be; Fondation Roi Baudouin, Faire face à la radicalisation violente : le soutien aux familles, Compte rendu des séminaires, Bruxelles, septembre 2015-janvier 2016 ; La Pensée et les Hommes, n91, Bruxelles, 2013 ; Caroline S GESSER, La fréquentation des cours de religion et de morale après l'introduction du cours de philosophie et de citoyenneté ", Les analyses du CRISP en ligne, Bruxelles, 8 avril 2019, www.crisp.be.

39. Existe aussi un appel à projets annuel sur " Promotion de la citoyenneté et de l'interculturalité " proposé par la Fédération Wallonie-Bruxelles et qui est destiné au secteur associatif et des pouvoirs publics locaux. Il vise à " soutenir des actions de sensibilisation, d'éducation et de participation à la vie sociale et politique porteuses d'égalité sociale, d'émancipation individuelle et de solidarité ". Trois axes sont déclinés : éducation des jeunes à la citoyenneté, promotion du dialogue interculturel et la prévention du racisme, protection et promotion des droits des personnes migrantes, en particulier le droit des femmes ".

40. Le ministère de la Fédération Wallonie-Bruxelles (FWB) a formé deux commissions, chacune composée de plus de 25 experts provenant du monde politique, universitaire, des organisations citoyennes, du corps enseignant et d'inspection. L'enseignement CPC concerne l'enseignement organisé par la Fédération Wallonie-Bruxelles (W-B E), l'enseignement officiel subventionné (CECP) et l'enseignement libre non confessionnel subventionné. 
41. Dans l'enseignement fondamental et le programme du secondaire ( $1^{\text {er }}$ degré), le programme inclut plusieurs chapitres à enseigner : construire une pensée autonome critique (1), se connaître soi-même et s'ouvrir aux autres (2), Construire la citoyenneté dans l'égalité en droits et en dignité (3), S'engager dans la vie sociale et l'espace démocratique (4). Relevons que dans le fondamental, le programme intègre deux fiches pédagogiques relatives à des situations particulières à savoir : " L'école, l'équipe, l'enseignant face à un évènement direct, brutal, tragique " et "L'école, l'équipe, l'enseignant face à la violence et à ses différentes formes (harcèlement, intimidation, humiliation...) ». Dans l'enseignement secondaire ( $2^{\mathrm{e}} / 3^{\mathrm{e}}$ degré), le programme intègre plusieurs thématiques dont " Discours et pièges du discours ", "Stéréotypes, préjugés et discriminations ", "Participer au processus démocratique ", "Légitimité et légalité de la norme ", "Diversité des discours sur le monde ", "Violence et humanisation ", "Rapport éthique à soi et à autrui ", "Vérité et pouvoir ", " Liberté et responsabilité ", " Participer au processus démocratique ", "Culture(s) et liberté(s) ", "Conviction, religion, politique ", etc. 42. A propos des détails propositionnels sur les pistes de travail et les expériences autour du désengagement, cf. Dounia BOUZAR, Etapes du processus de radicalisation et de déradicalisation, Practicies, projet de recherche, mai 2018, pp. 153 et sv. ; Vincent SERON, Prévention de la radicalisation : aperçu des différents modèles utilisés à l'échelle internationale, CETR, ULg, 27 avril 2018 (document non accessible) ; Jean-Marie BOCKEL et Luc CARVOUNAS, Rapport d'information $n^{\circ} 483$ sur les collectivités territoriales et la prévention de la radicalisation, Sénat, Paris, 29 mars 2017 ; Esther BENBASSA et Catherine TROENDLE, Rapport d'information $n^{\circ} 633$ sur le désendoctrinement, le désembrigadement et la réinsertion des djihadistes en France et en Europe, Sénat, Paris, 12 juillet 2017. 


\section{Sécurité Globale \\ Bulletin d'abonnement ou de réabonnement}

À retourner accompagné de votre règlement aux

Éditions ESKA - 12, rue du Quatre-Septembre, 75002 PARIS

Tél. : 0142865565 - Fax : 0142604535

M, Mme, Mlle Prénom

Société/Institution

$\mathrm{N}^{\circ}$

Rue

Code postal Ville

Pays

Adresse électronique

TARIFS D'ABONNEMENTS*

\begin{tabular}{|l|c|c|c|c|}
\hline & $\begin{array}{c}\text { France } \\
\text { particulier }\end{array}$ & $\begin{array}{c}\text { France } \\
\text { société/ } \\
\text { institution }\end{array}$ & $\begin{array}{c}\text { Etranger } \\
\text { particulier }\end{array}$ & $\begin{array}{c}\text { Etranger } \\
\text { société/ } \\
\text { institution }\end{array}$ \\
\hline 1 an (2020) & $\square 111 €$ & $\square 141 €$ & $\square 136 €$ & $\square 167 €$ \\
\hline 2 ans (2020 et 2021) & $\square 200 €$ & $\square 250 €$ & $\square 240 €$ & $\square 299 €$ \\
\hline
\end{tabular}

* Abonnements souscrits à l'année civile (janvier à décembre).

Je souscris un abonnement pour $\quad \square 1$ an $\quad \square 2$ ans

Je joins mon règlement de Euros

$\square$ par chèque bancaire à l'ordre des Éditions ESKA

- par virement bancaire aux Éditions ESKA - BNP Paris Champs Elysées 30004/00804/ compte : 0001013985836

$\square$ par carte bancaire : merci d'indiquer votre numéro de compte et la date d'expiration $\mathrm{N}^{\circ}$ carte bancaire: $\square$ Visa $\square$ Eurocard/Mastercard

Date d'expiration :

Signature :

\section{Derniers numéros parus}

Sécurité globale 19 | 2019 (nouvelle série) : ANSSI. 10 anniversaire - " Diversité ». Un mot sans histoire Sécurité globale 18 | 2019 (nouvelle série) : Maîtrise du terrorisme par le haut Sécurité globale $17 \mid 2019$ (nouvelle série) : Géopolitique, Sécurité-Légalité Sécurité globale 16 2018 (nouvelle série) : Brésil demain : Sécurité, économie, écologie Sécurité globale 15 | 2018 (nouvelle série) : Cybermonde : état des lieux, perspectives, risques et périls Sécurité globale 14 | 2018 (nouvelle série) : Géopolitique - Terrorismes et crime organisé Sécurité globale $13 \mid 2018$ (nouvelle série) : Terrorisme - Criminologie Sécurité globale $12 \mid 2017$ (nouvelle série) : Terrorisme - Criminologie Sécurité globale 11 | 2017 (nouvelle série) : Géopolitique - Criminologie - Terrorisme Sécurité globale 10 | 2017 (nouvelle série) : Le chi'isme paramilitaire Sécurité globale 9 | 2017 (nouvelle série) : Les habits neufs de l'impérialisme Sécurité globale 8 | 2016 (nouvelle série) : Cyber-chaos et sécurité numérique Sécurité globale $7 \mid 2016$ (nouvelle série) : Islam activiste, réaction et révolution Sécurité globale 6 | 2016 (nouvelle série) : Le monde criminel à l'horizon 2025 Sécurité globale $5 \mid 2016$ (nouvelle série) : Dossier Stupéfiants Sécurité globale 3-4 | 2015 (nouvelle série) : Toujours plus cyber-menacées : les collectivités territoriales / "Police prédictive " : les belles histoires de l'Oncle Predpol Sécurité globale 2 | 2015 (nouvelle série) : Bandes, Braquages, Terreur

\section{ÉDITIONS ESKA}

